\title{
A microfabricated electroosmotic pump coupled to a gas-diffusion microchip for flow injection analysis of ammonia
}

\author{
Zaifang Zhu' ${ }^{1}$ Joann J Lu${ }^{1}$, M. Inês G. S. Almeida ${ }^{2}$, Qiaosheng $\mathrm{Pu}^{3}$, Spas D. Kolev², Shaorong Liu ${ }^{1}$ \\ ${ }^{1}$ Department of Chemistry and Biochemistry, University of Oklahoma, Norman, OK 73019, USA, e-mail: \\ shaorong.liu@ou.edu \\ ${ }^{2}$ School of Chemistry, The University of Melbourne, Victoria 3010, Australia, e-mail: s.kolev@unimelb.edu.au \\ ${ }^{3}$ College of Chemistry and Chemical Engineering, Lanzhou University, Lanzhou, Gansu 730000, P.R. China
}

\begin{abstract}
Flow injection analysis (FIA) is an analytical technique capable of performing multiple sample pretreatment processes. Theoretically, most FIA methods can be implemented on lab-on-chip devices. However, not many FIA applications have been carried out using lab-on-chip devices, presumably due to the lack of some basic functional components (e.g., pumps, valves, detectors). We have microfabricated two functional components toward developing a microchip FIA system, i.e., an open-channel electroosmotic pump and a gas-diffusion chip, consisting of two microfabricated glass wafers and a porous polytetrafluoroethylene membrane. This is the first application of gas-diffusion separation in a microchip FIA system. To demonstrate the feasibility of using these two components for performing gas-diffusion FIA, we have incorporated them together with a regular FIA injection valve and a capillary electrophoresis absorbance detector in a flow injection system for measuring ammonia in environmental water samples. This system has a limit of detection of $0.10 \mathrm{mg} \mathrm{L}^{-1} \mathrm{NH}_{3}$, with a good repeatability (relative standard deviation of less than $5 \%$ for $4.0 \mathrm{mg} \mathrm{L}^{-1} \mathrm{NH}_{3}$ ). Parameters affecting its performance are also discussed.
\end{abstract}

Keywords: microchip, microfluidic device, flow injection analysis, gas-diffusion, ammonia

Running title: microfabricated electroosmotic pump coupled to a gas-diffusion microchip

\section{Introduction}

The objective of lab-on-chip (LOC) is to integrate and perform multiple analytical processes (e.g., sample pretreatment, solution distribution/mixing, separation, and detection) on a microchip platform. A lot of research has been focused on electrophoretic separations, and relatively speaking, much less work has been carried out on multi-process integration, likely due to the lack of some robust and miniaturized functional components (e.g., pump, valve) that can be readily incorporated into an LOC device. Flow injection analysis (FIA), developed in the mid-1970s [1], is an analytical technique capable of performing multiple and complex sample pretreatment processes. So far, only a few FIA applications have been implemented using LOC devices.

Even fewer LOC devices have been implemented in environmental analysis [2,3]. The reasons might be that environmental samples are abundant, and that the reagents used for these analyses are relatively inexpensive compared to scarce samples and expensive reagents for some bioanalyses. However, LOC devices can bring quite a few benefits to environmental analysis [4]. For example, LOC devices are miniaturized and portable, and can be conveniently taken to the field for on-site analysis. Low-cost and miniaturized LOC devices can be placed at multiple sites for unattended monitoring. LOC devices are generally environmentally friendly because these systems consume small quantities of 
reagents and produce low volumes of wastes.

Gas-diffusion (GD) devices are commonly used to improve analysis selectivity by separating a volatile or semi-volatile analyte or analyte that can be converted into a volatile species from its interferents in the sample matrix [5]. A GD device often consists of a hydrophobic porous membrane and two fluidic conduits on both sides of the membrane, i.e. donor and acceptor channels. As the sample is brought by donor stream to the membrane, the analyte or its volatile derivative evaporates into the membrane pores, diffuses across the porous membrane, and is absorbed by the acceptor solution on the other side of the membrane for further processing and detection. The GD device can be conveniently integrated with an FIA or sequential injection analysis (SIA) system [6-8], and it offers an effective means of avoiding interferences and thus minimizing matrix effects.

Ammonia, present in nature as neutral ammonia and the ammonium cation, is an excellent source of plant nitrogen. It is either of natural (e.g., formed during decomposition of proteins and other nitrogen-containing compounds) or anthropogenic origin (e.g., released with industrial, domestic or agricultural effluents). Low-level ammonia is not considered toxic to humans; the US National Academy of Science recommends, and many European nations have adopted, a drinking water standard of $0.5 \mathrm{mg} \mathrm{L}^{-1}$. Some drinking water treatment processes add small amounts of ammonia, generally less than $0.4 \mathrm{mg} \mathrm{L}^{-1}$ to water to increase and extend the disinfecting ability of chlorine [9]. Ammonia has a toxic effect on humans if the intake becomes higher than the capacity to detoxify it, e.g., at a dose of more than $33.7 \mathrm{mg}$ of ammonia per kg of body mass per day; ammonia influences metabolism by shifting acid-base equilibria, disturbing the glucose tolerance, and reducing the tissue sensitivity to insulin [10]. Ammonia is also toxic to fish and other aquatic organisms often at concentrations even below $1 \mathrm{mg} \mathrm{L}^{-1}$ in water.

For measuring the concentration of ammonia in a water sample using a GD device, the donor solution is usually alkaline to convert quantitatively the ammonium cation to ammonia, the acceptor solution contains often an acid-base indicator that changes color after absorbing ammonia, and the hydrophobic porous membrane is frequently made of polytetrafluroethyelene (PTFE). Such membranes transport ammonia and other gaseous compounds and prevent solutes from being exchanged between the donor and acceptor solutions $[6,7,11,12]$. In a GD-FIA system, the donor and acceptor solutions are propelled to the membrane via a multichannel peristaltic pump, the sample is injected into the donor stream via an injection valve, and the quantity of the ammonia is measured usually by measuring the color change of the acceptor stream.

In this study, we microfabricated two basic functional components toward the ultimate development of a microchip GD-FIA system. We microfabricate an electroosmotic pump (EOP) following a similar process as previously described by us [13-15] but with some modifications. We assembled for the first time a GD chip using two glass wafers with microfabricated channels and a porous PTFE tape. We incorporated the EOP and the GD chip with a conventional FIA injection valve and a CE absorbance detector, constituting a partially-microfabricated GD-FIA system. We characterized this GD-FIA system and utilized it for measuring ammonia concentrations in environmental water samples to demonstrate the feasibility of using the two microfabricated components for performing FIA.

\section{Experimental}

\section{Reagents and Materials}

Ammonium chloride, bromothymol blue (BTB), sodium tetraborate decahydrate (borax), phenol, sodium nitroprusside, ethylenediaminetetraacetic acid (EDTA), hydrochloric acid and sodium 
hydroxide were purchased from ThermoFisher Scientific (Waltham, MA, http:/www.thermofisher.com/en/home.html). Sodium hypochlorite (Clorox, http://www.thecloroxcompany.com/, $5 \% \mathrm{w} / \mathrm{v} \mathrm{NaOCl)} \mathrm{was} \mathrm{obtained} \mathrm{from} \mathrm{a} \mathrm{local} \mathrm{Walmart.} \mathrm{The} \mathrm{donor}$ solution contained 1.0 M sodium hydroxide + 0.10 M EDTA, and the acceptor solution contained 0.060 $\mathrm{g} \mathrm{L}^{-1} \mathrm{BTB}$ with its $\mathrm{pH}$ being adjusted to 5.9 using hydrochloric acid. The pumping solution was 2.0 $\mathrm{mM}$ Borax $(\mathrm{pH}=9.2)$. The pumping solution was ultrasonically degassed daily before use. Microposit S1818 photoresist, MF-319 developer, Chrome etchant, and gold etchant were purchased from Shipley Company (Marlborough, MA). The porous PTFE membrane (BridglandTM standard white Teflon tape) was purchased from Reece Australia Limited (VIC, Australia, http://www.reece.com.au/). Fused silica capillaries were bought from Molex Inc. (Phoenix, AZ, http://www.molex.com/molex/index.jsp). Borofloat glass wafers were bought from Precision Glass \& Optics (Santa Ana, CA). Nafion membrane 115 was from Fuel Cell Earth, LLC (Stoneham, MA, http://fuelcellearth.com/). All solutions were prepared in deionized water (Millipore, Synergy 185, France, resistivity $>18.2 \mathrm{M} \Omega \mathrm{cm}$, http://www.merckmillipore.com/INTL/en).

\section{EOP construction}

Figure 1A schematically presents the EOP channel pattern. The pattern for all connection-channels (red) and alignment-marks (blue) were laid out on Photomask \#1 and had a linewidth of $10 \mu \mathrm{m}$, while the pattern for all pump channels (green) were placed on Photomask \#2 and had a linewidth of $300 \mu \mathrm{m}$. All connection channels were semicircles, except for the straight channels leading to the edge of the wafer. All pump channels had an identical length of $60 \mathrm{~mm}$, and there were six repetitive channel groups in this channel pattern design. Within each channel group, the center-to-center distance between two adjacent pump channels was $450 \mu \mathrm{m}$. The fabrication process was similar to what we had described previously $[15,16]$. Briefly, $\sim 190-\mu$ m-deep connection-channel grooves were produced on two 98-mm-diameter glass wafers, first using Photomask \#1. Because of the isotropic etching of HF, these grooves had a semicircular profile with an i.d. of $\sim 380 \mu \mathrm{m}$ that matched accurately the o.d. (375 $\mu \mathrm{m})$ of the connection capillaries. Photomask \#2 was then used to align the pump channel pattern with the etched connection-channel grooves on one of the above wafers, and etched to a depth of $\sim 5 \mu \mathrm{m}$. After 1-mm-diameter access holes were drilled through the connection-channel grooves on the wafer with only the connection-channel grooves, two wafers were cleaned, face-to-face aligned, and thermally bonded. The resultant chip had round connection channels with a diameter of $\sim 380 \mu \mathrm{m}$ and flat pump channels with a depth of $\sim 5 \mu \mathrm{m}$ and width of $\sim 310 \mu \mathrm{m}$. The chip was cut to a desired shape, capillaries were inserted into the connection channels, and adhesive was applied to the access holes to secure the capillaries. Figure 1B presents an image of an EOP chip with capillaries being attached. (Note: the two short-pump-channel EOP units were not used in this experiment.) Figure 1C presents a Nafion membrane grounding interface for the EOP. A micro cross was utilized to incorporate a Nafion membrane into the fluidic system to facilitate application of an electric potential to the pump channels. At each end of the cross there was a threaded hole with a flat bottom. A piece of PTFE tubing with an inner diameter (i.d.) of $0.8 \mathrm{~mm}$ was inserted through a nut to the flat end of a ferrule (Fig. 1C). A piece of Nafion membrane 115 (Fuel Cell Earth, LLC, Stoneham, MA) was cut to an appropriate size, hydrated first in ethanol and then in $2.0 \mathrm{mM}$ borax solution, and put to the bottom of a threaded hole. By tightening the nut, the ferrule pressed the Nafion membrane tightly against the flat bottom of the threaded hole. Although the Nafion membrane blocked the liquid flow, cations could pass across the 
this cation exchange membrane easily. The other three ends were used for incorporating connection capillaries. Figure 1D presents a schematic configuration of the EOP; two channel groups are combined into one EOP via a micro cross. During the pump operation, a positive HV electrode and the loose ends of the inlet connection capillaries were dipped into a $500-\mathrm{mL}$ borax reservoir, while a grounding electrode (GND) and the loose ends of the PTFE tubings were dipped into another 500-mL borax solution container.

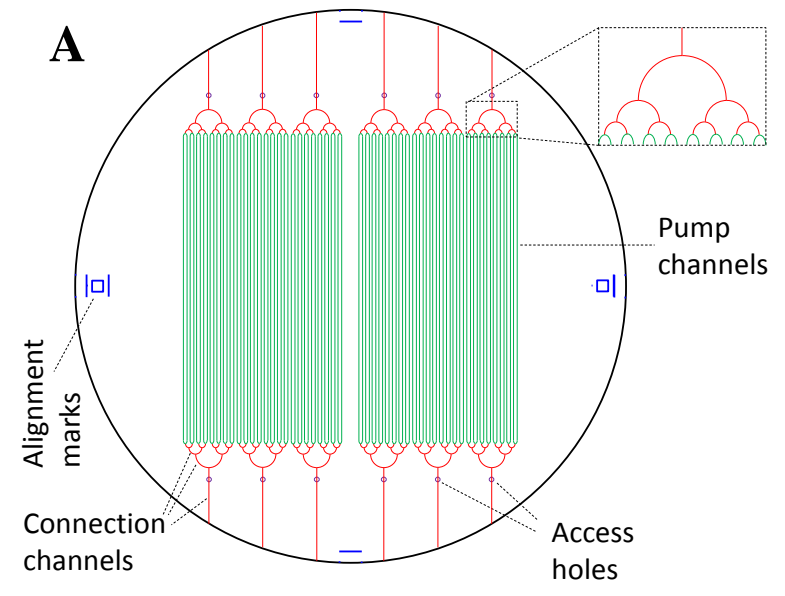

C

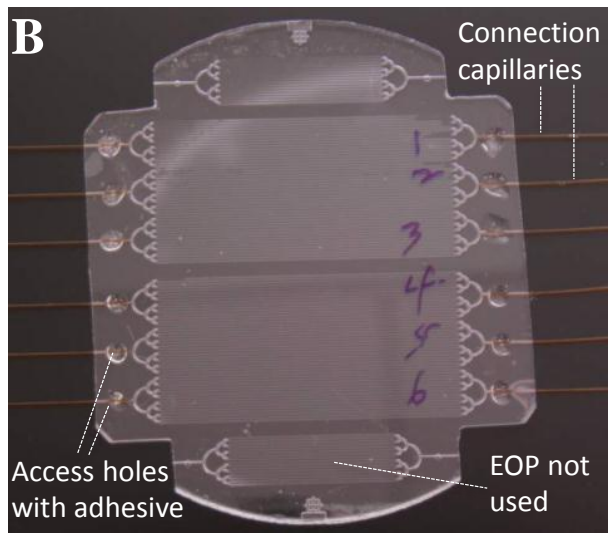

D

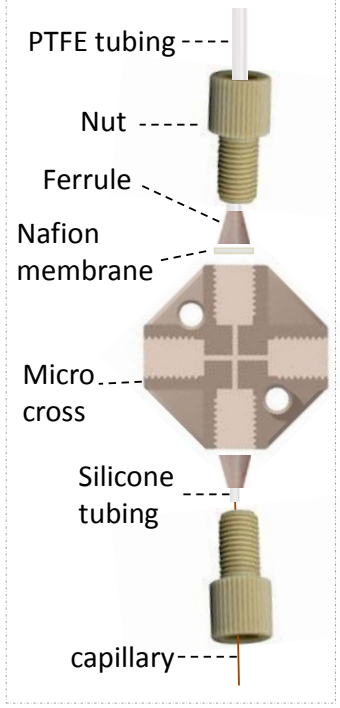

\section{D}

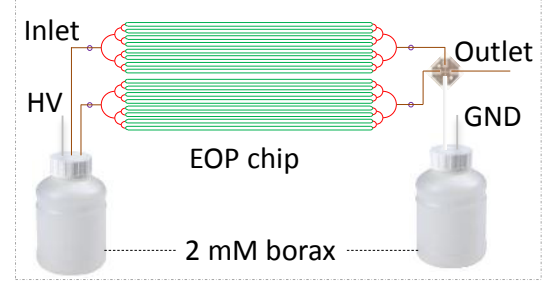

Figure 1. EOP chip. (A) EOP channel pattern. Pump channel dimensions: 60 -mm-long $\times 310-\mu m$-wide $\times$ 5- $\mu \mathrm{m}$-deep; connection channel diameter: $\sim 380 \mu \mathrm{m}$; Access-hole: 1-mm-diameter. (B) EOP chip. Connection capillary i.d.: $250 \mu \mathrm{m}$. (C) Nafion membrane grounding interface. PTFE tubing i.d.: $0.8 \mathrm{~mm}$; capillary i.d.: 250 $\mu \mathrm{m}$. (D) Schematic configuration of microchip EOP. Three channel groups (only two are shown here) and two crosses (only one is shown here) were used to construct one EOP; HV: $4 \mathrm{kV}$; pump solution container volume:

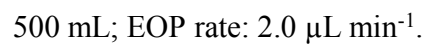

\section{Manufacturing of the gas-diffusion chip}

Figure 2A schematically presents a photomask design containing five different channel geometries $\left(1-1^{\prime}, 2-2^{\prime}, 3-3^{\prime}, 4-4^{\prime}\right.$, and 5-5'). All geometries had the same photomask linewidth of $100 \mu \mathrm{m}$. The distance between the folded channels or parallel channels (from center-to-center) was $1 \mathrm{~mm}$. After all channel patterns were etched on a wafer to a depth of $\sim 50 \mu \mathrm{m}$, holes were drilled at all ends of the grooves, and the wafer was then cut vertically (along channel geometry 5-5') into two halves. [Note: To obtain a chip for testing channel 5-5', the wafer was cut horizontally through the middle of all the other 
grooves.] The two halves were cleaned and face-to-face aligned to sandwich a piece of porous PTFE membrane, and the assembly was held together using 4 paper clips (Fig. 2B). Figure $2 \mathrm{C}$ presents the channel orientation after the GD chip was assembled. [Note: The red channel on the bottom plate should be invisible because it was underneath the PTFE membrane. It is shown in Fig. 2C for illustration purposes only.] The combination of Fig. 2C and 2D depicts fluidic conduits thoroughly. A capillary was plugged into every 1-mm-diameter access holes via a pipette tip sleeve, and secured in position using epoxy adhesive. Figure 2E shows a picture of an assembled GD chip (with the paper clips removed). [Note: The red plastic block was not part of the GD device, and it was used to support the GD device for taking the picture.]
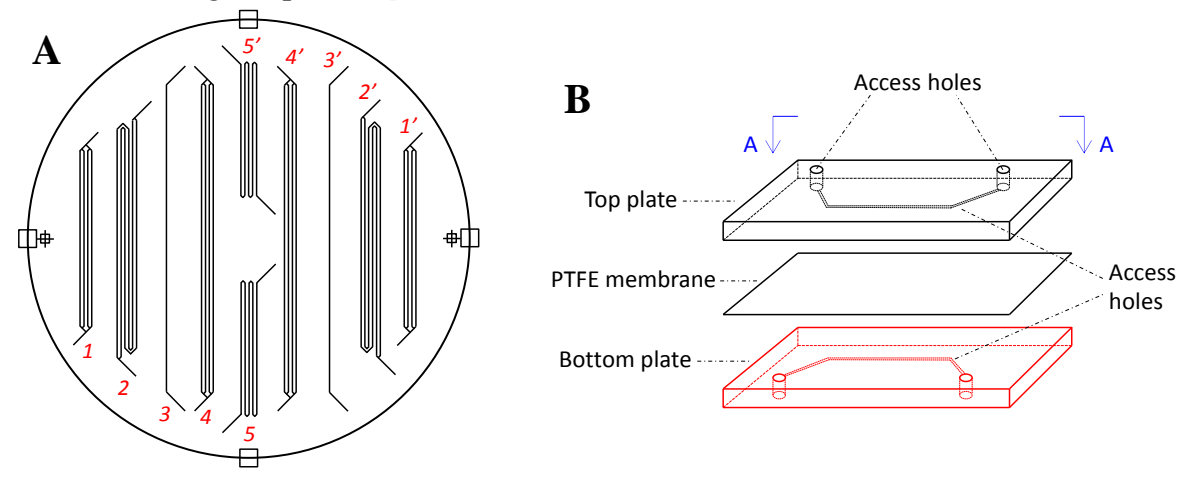

\section{$\mathbf{E}$}
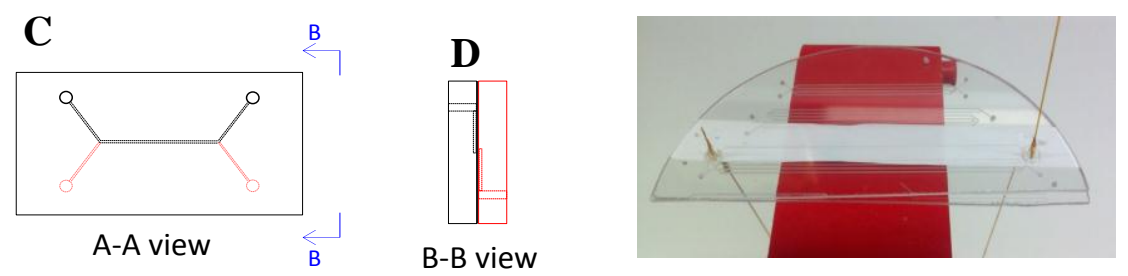

Figure 2. GD chip. (A) Channel geometry. Channel depth and width: $50 \mu \mathrm{m}$ and $140 \mu \mathrm{m}$; effective channel lengths: 4, 15.5, 7, 7, and $15 \mathrm{~cm}$ for 1-1', 2-2', 3-3', 4-4', and 5-5', respectively. (B) Schematic configuration of GD chip. (C) A-A view referenced to (B). (D) B-B view referenced to (C). (E) Picture of GD chip. All capillaries connected to GD chip had an i.d. of $250 \mu \mathrm{m}$. Paper clips were used to hold the GD chip together, but the picture was taken after paper clips were removed.

\section{Apparatus}

Figure 3 presents a schematic diagram of the apparatus used in these experiments. Figure 3B represents the $2.0 \mathrm{mM}$ borax pump solution. Two EOPs (EOP1 and EOP2) had identical configurations; each consisted of three pump-channel groups and had a flow rate of $2.0 \mu \mathrm{L} \mathrm{min}{ }^{-1}$ when $4 \mathrm{kV}$ was applied to the pump channels. The top fluidic line (blue) represents the acceptor stream, while the bottom line (red) represents the donor stream. Two reagent holding coils ( $\mathrm{HC1}$ and $\mathrm{HC} 2$ ) were incorporated to prevent the donor and acceptor solutions from contaminating the pump channels. The samples and standards were injected into the donor-stream using a Rheodyne Model 5020 rotary injection valve (V) with a sample-loop volume of $\sim 5 \mu \mathrm{L}$. The sample mixed with the donor solution and the ammonium cation was quantitatively converted to ammonia. When the donor stream passed through the GD chip, ammonia diffused across the PTFE membrane (M) and was absorbed by the acceptor solution containing BTB where it was converted back to the ammonium cation thus changing the solution $\mathrm{pH}$. 
This $\mathrm{pH}$ change resulted in a BTB color change that was measured by a capillary electrophoresis (CE) absorbance detector (D) (Linear UVIS 200, Linear Instruments, Reno, NV, USA, http://www.linearinstrument.com/) at $620 \mathrm{~nm}$. The absorbance signal was acquired using a NI multifunctional card DAQ Card (6062E, National Instruments, Austin, TX, http://www.ni.com/), and the data were processed with an in-house written LabView program.

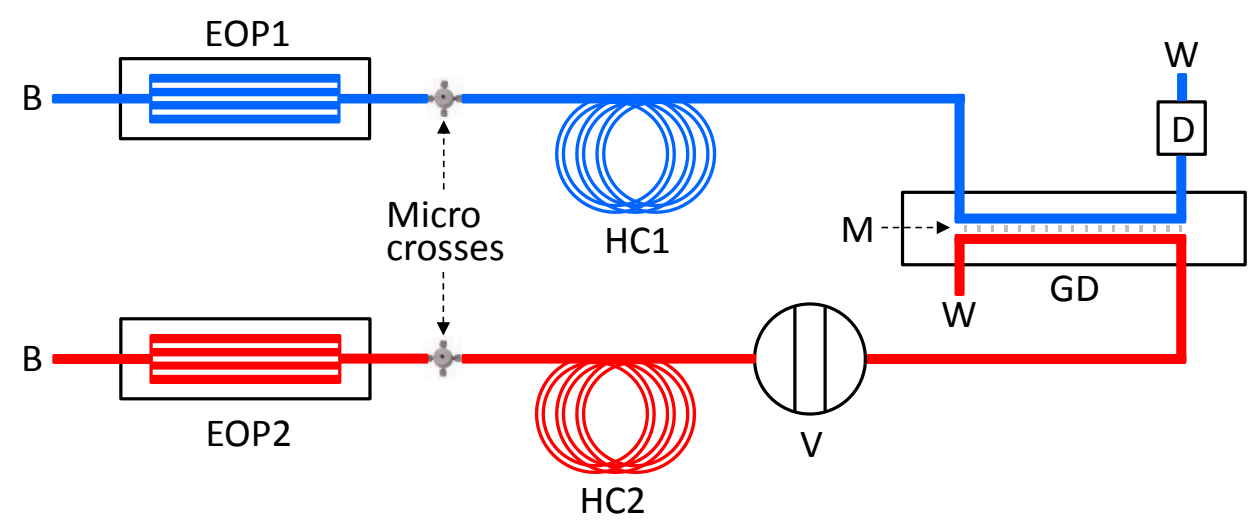

Figure 3. Schematic arrangement of the GD-FIA system. B: $2.0 \mathrm{mM}$ borax; $\mathrm{HC} 1$ and $\mathrm{HC} 2$ : reagent holding coils 1 and 2 , each with $\sim 250 \mu \mathrm{L}$ volume; V: FIA injection valve with a sample loop volume of $\sim 5 \mu \mathrm{L}$; M: Porous PTFE membrane; W: waste; GD: gas-diffusion chip; D: CE absorbance detector (620 nm).

\section{Berthelot method for ammonia measurement}

A Berthelot protocol, utilized earlier by us [17], was employed for determining ammonia in natural samples in order to validate the accuracy of the method. Phenol/sodium nitroprusside solution (R1) was prepared by dissolving $1.0 \mathrm{~g}$ phenol and $0.5 \mathrm{~g}$ sodium nitroprusside in $50 \mathrm{~mL}$ of deionized water. Alkaline EDTA reagent solution (R2) was made by dissolving $5.0 \mathrm{~g}$ disodium ethylenediaminetetraacetate-hydrate and $2.0 \mathrm{~g}$ sodium hydroxide in $100 \mathrm{~mL}$ of deionized water. Sodium hypochlorite reagent solution (R3) was obtained by diluting $8 \mathrm{~mL}$ commercial bleach (Clorox, $5 \% \mathrm{w} / \mathrm{v} \mathrm{NaOCl}$ ) with deionized water to $100 \mathrm{~mL}$. To perform the measurement, $5.0 \mathrm{~mL}$ of R2, $5.0 \mathrm{~mL}$ of R3, and $5 \mathrm{~mL}$ of R1 were successively added into a $50-\mathrm{mL}$ flask containing $25.0 \mathrm{~mL}$ of sample or standard (for creating a calibration curve) and the mixture was diluted to $50 \mathrm{~mL}$ with deionized water. The solution was mixed gently and left at ambient temperature $\left(\sim 20{ }^{0} \mathrm{C}\right)$ for $\sim 30$ min and then the absorbance was measured using a Shimadzu UV-1601 UV-Visible Spectrophotometer (http://www.shimadzu.com/) at $630 \mathrm{~nm}$. The transient absorbance peak maximum was used as the analytical signal in this study.

\section{Results and discussion}

\section{General considerations regarding system design and assembling}

The primary purpose of this work was to microfabricate two functional components (an EOP and a GD chip) for the construction of a cost-effective microfluidic GD-FIA system, and to demonstrate the feasibility of using this system for natural water sample analysis. Preparation of a microchip EOP had been reported before [14-16], but improvements were made in this work. The major improvement was the Nafion membrane grounding interface. The interface allowed application of potential efficiently on 
the pump channels. Because the fully-hydrated Nafion 115 membrane had a low electric resistivity $(10-12 \Omega \mathrm{cm})$ [18], and because the PTFE connection tubing was short $(\sim 5 \mathrm{~cm})$ and wide $(0.8 \mathrm{~mm}$ i.d.), most of the voltage (>99\%) was effectively applied to the pump channels. The interface was robust, and the EOP worked smoothly.

Figure 3 presents a schematic arrangement of the microfluidic GD-FIA system. Reagent holding coils (HC) were incorporated between an EOP and the GD chip using micro unions. $\mathrm{HC} 1$ and HC2 were two 5-m-long and 250- $\mu \mathrm{m}$-i.d capillaries, with $\mathrm{HC} 1$ holding $0.060 \mathrm{~g} \mathrm{~L}^{-1} \mathrm{BTB}(\mathrm{pH}=5.9)$ and $\mathrm{HC} 2$ holding $1.0 \mathrm{M} \mathrm{NaOH}+0.10 \mathrm{M}$ EDTA. As the EOPs were turned on, they pumped $2.0 \mathrm{mM}$ borax solution into the holding coils, pushed the reagents in $\mathrm{HC} 1$ and $\mathrm{HC} 2$ through the GD chip. As can be seen, the donor and acceptor streams flew in a counter-current format. Because the GD channels were narrow $(\sim 200 \mu \mathrm{m})$, they supported the PTFE membrane well, enabling us to run GD in a counter-current format. In conventional GD devices, the width of the GD channels is often around 2 $\mathrm{mm}$ [8], and a counter-current flow could distort the membrane due to the pressure differences between the donor stream on one side and the acceptor stream on the other side of the PTFE membrane.

\section{Ammonia collection efficiency}

Figure 2A presents the photomask with various line patterns. Pattern $3 / 3^{\prime}$ had the simplest geometry; a single straight GD channel (Fig. 2C). Pattern 5/5' had a single but folded channel to extend the GD channel length; we hypothesized that an extended GD channel would improve the ammonia collection efficiency by the acceptor solution. Pattern $2 / 2^{\prime}, 1 / 1^{\prime}$ and $4 / 4^{\prime}$ had parallel channels; we hypothesized that parallel GD channels would improve the ammonia collection efficiency and consequently the detection sensitivity. Our test results revealed that changing the channel patterns had increased the analytical signal by less than $10 \%$.

We also assessed the collection efficiency of the GD chip by stopping both the donor and acceptor streams after a sample zone entered the GD donor channel. We hypothesized that, if the collection efficiency under flow conditions was not the maximum possible one, an increased amount of ammonia should be transferred across the PTFE membrane when the solutions were stopped and this amount should increase with increasing the duration of the stop-flow time. The results showed that the analytical signals did not increase by more than $10 \%$ for different stop-flow times, indicating that the collection efficiencies in the case of both counter-current flow and stop-flow conditions corresponded to more than $90 \%$ transfer of ammonia from the donor to the acceptor channel of the GD chip. Therefore, pattern $3 / 3^{\prime}$ was selected for use in the subsequent experiments because of its simplicity and the fact that it produced the smallest error bar.

The 50- $\mu \mathrm{m}$-deep GD channel could have contributed significantly to achieving maximum ammonia collection efficiency both under counter-current flow and stop-flow conditions. The GD channels had an effective length (the straight portion of the channel) of $\sim 70 \mathrm{~mm}$ and an average width of $\sim 140 \mu \mathrm{m}$, thus having an effective volume of $\sim 0.5 \mu \mathrm{L}$. In these experiments, EOP1 and EOP2 had similar pump rates, $\sim 2.0 \mu \mathrm{L} \mathrm{min}{ }^{-1}$. This led to an average time of $t=\sim 15 \mathrm{~s}$ for the sample zone to spend inside the GD channel. In an aqueous solution at room temperature, ammonia has a diffusion coefficient of $\mathrm{D}=$ $1.5 \times 10^{-9} \mathrm{~m}^{2} \mathrm{~s}^{-1}$ [19]. During the 15-s period, ammonia could have diffused to an average distance of $\sim 150 \mu \mathrm{m}(=\sqrt{D \cdot t})$, three times the depth of the GD channel. In conjunction with the flow dispersion, most of the ammonia molecules would have diffused across the PTFE membrane and be absorbed by the acceptor solution. Therefore, the channel width is not expected to have influenced significantly the ammonia collection efficiency. 


\section{Effect of injection time on the analytical signal}

Because the injection valve was inserted directly into the donor-solution line, it was expected that the length of the sample plug would affect ammonia-NaOH mixing. The injection valve had a sample-loop volume of $\sim 5 \mu \mathrm{L}$, which would occupy $\sim 10 \mathrm{~cm}$ of the $250-\mu \mathrm{m}$-i.d. donor-solution line if the sample was fully injected. We normally injected only a small portion of the sample into the donor-solution line by controlling the injection time. Figure 4 presents the effect of injection time on absorbance signal. Since the pump had a flow rate of $2.0 \mu \mathrm{L} \mathrm{min}{ }^{-1}$, the injection time of $5(10,15,20,25$, or 30$) \mathrm{s}$ corresponded to an injected volume of $0.17(0.33,0.50,0.67,0.83$, or 1.0$) \mu \mathrm{L}$. The full-injection indicated all the sample in the sample-loop was injected into the donor-solution line. As can be seen from Fig. 4, the absorbance signal increased with injection time, and plateaued around 25 30 s. However, because double-humped peaks appeared after $20 \mathrm{~s}$, we used $15 \mathrm{~s}$ injection time for the rest of the experiments.

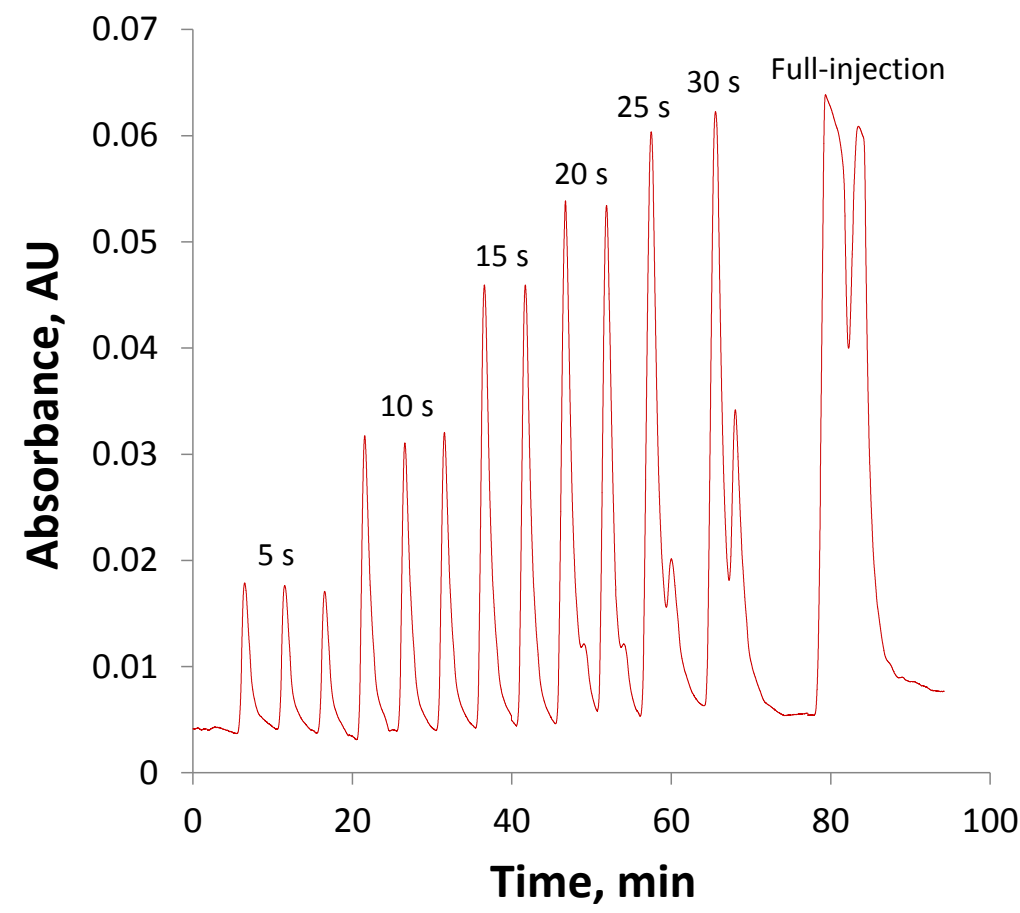

Figure 4. Effect of injection time on the analytical signal. GD channel: 3-3'; sample: $4.0 \mathrm{mg} \mathrm{L}^{-1} \mathrm{NH}_{3}$; wavelength: $630 \mathrm{~nm}$.

\section{Analytical figures of merit}

Fig. 5A presents the calibration curve for the GD-FIA system. Good linear relationship $\left(\mathrm{R}^{2}=0.999\right)$ was obtained between the analytical signal (maximum absorbance) and ammonia concentration in the range from $0.25 \mathrm{mg} \mathrm{L}^{-1}$ to $5 \mathrm{mg} \mathrm{L}^{-1}$. Figure $5 \mathrm{~B}$ illustrates the good repeatability of the method. The ten replicate runs of $4.0 \mathrm{mg} \mathrm{L}^{-1} \mathrm{NH}_{4}{ }^{+}$standard yielded a relative standard deviation of $4.8 \%$. Using the standard deviation of five blank-injections as the noise, the limit of detection of this method was calculated to be $\sim 0.10 \mathrm{mg} \mathrm{L}^{-1}$ ( $\mathrm{S} / \mathrm{N}=3$ ). Under optimal conditions, a throughput of 12 samples per hour was achieved. Obviously, the sample throughput could be boosted by increasing the EOP rates. 

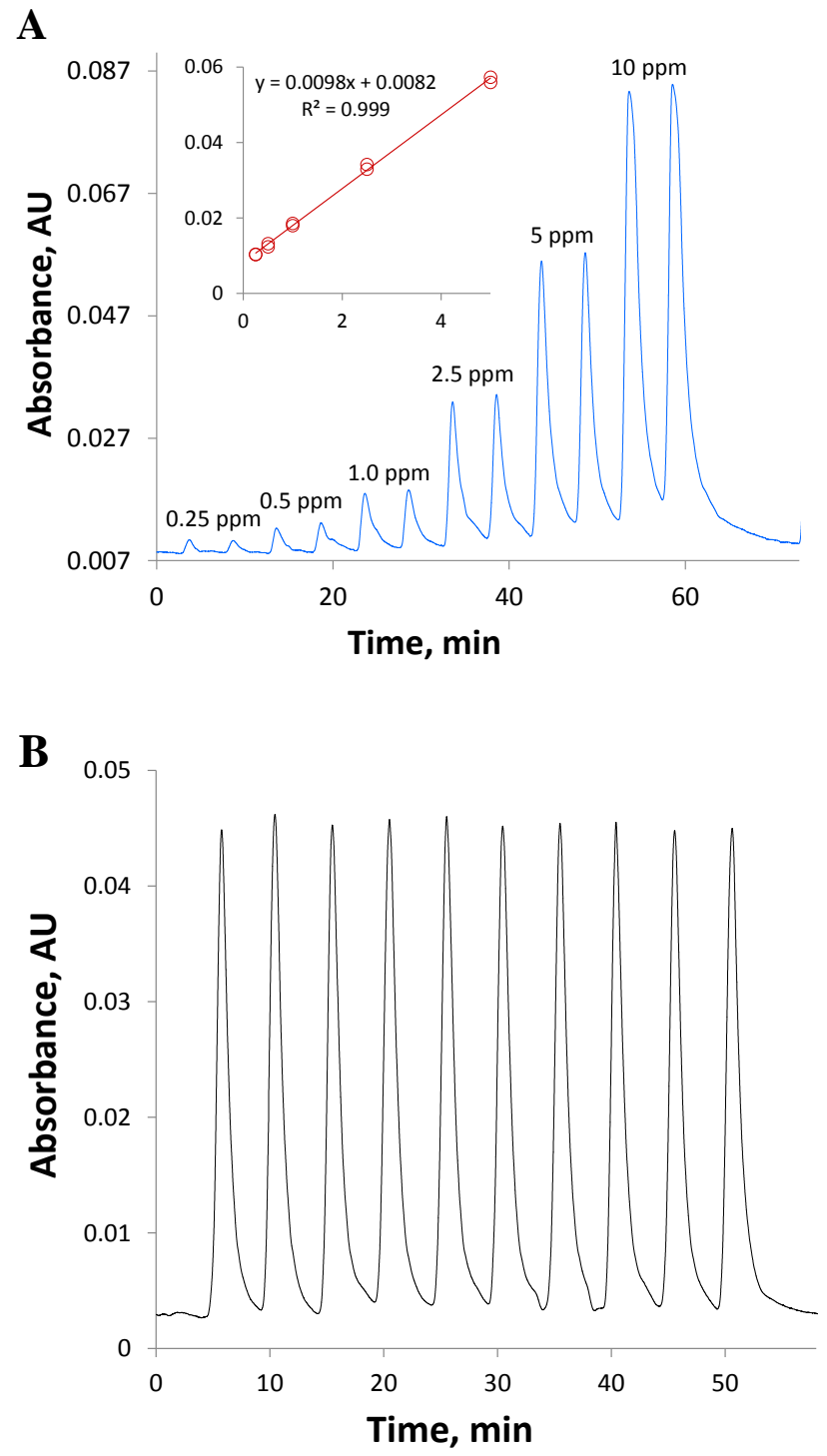

Fig. 5. GD-FIA calibration curve and replicate absorbance peaks. GD channel: 3-3'; injection time: 15 s; sample throughput: $12 \mathrm{~h}^{-1}$; wavelength: $630 \mathrm{~nm}$. (A) Calibration curve. inset: linear regression results for $\left[\mathrm{NH}_{3}\right]$ varying from $0.25 \mathrm{mg} \mathrm{L}^{-1}$ to $5.0 \mathrm{mg} \mathrm{L}^{-1}$. (B) Replicate absorbance peaks. Sample: $4.0 \mathrm{mg} \mathrm{L}^{-1} \mathrm{NH}_{4}^{+}$.

\section{Analysis of natural water samples}

Environmental water samples were collected from Lake Thunderbird in Norman, Oklahoma at three different sites and at two different depths in May/June of 2014. These samples were brought to the lab, filtered using a VWR $0.2-\mu \mathrm{m}$ membrane filter, and injected in triplicate into the GD-FIA system for ammonia measurement. The samples were also analyzed using Berthelot method. The experimental results presented in Table 1 show that the two methods were in good agreement. There was no statistical significant difference between the results obtained by the two methods at the $95 \%$ confidence limit. 
Table 1. Ammonia concentrations and the corresponding standard deviations for samples collected at different sites of Lake Thunderbird ( $\left.\mathrm{mg} \mathrm{L}^{-1}\right)$ determined by the developed GD-FIA method and the batch Berthelot method.

\begin{tabular}{|c|c|c|c|c|c|c|}
\hline & Site 1 & Site 1 & Site 2 & Site 2 & Site 3 & Site 3 \\
\hline source & On surface & 5-m-deep & On surface & 5-m-deep & On surface & 5-m-deep \\
\hline GD-FIA $^{a}$ & $0.60 \pm 0.03$ & $0.90 \pm 0.05$ & $0.55 \pm 0.02$ & $0.83 \pm 0.04$ & $0.62 \pm 0.03$ & $0.86 \pm 0.04$ \\
\hline $\begin{array}{l}\text { Berthelot } \\
\text { method }^{\mathrm{b}}\end{array}$ & $0.64 \pm 0.01$ & $0.88 \pm 0.03$ & $0.58 \pm 0.02$ & $0.86 \pm 0.02$ & $0.62 \pm 0.01$ & $0.82 \pm 0.03$ \\
\hline
\end{tabular}

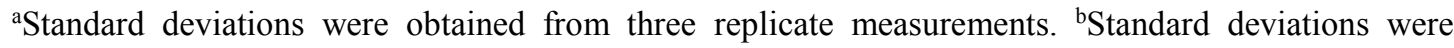
obtained from 5 replicate measurements.

\section{Conclusions}

We have microfabricated an EOP and a GD chip and incorporated these two functional components into a GD-FIA system. This is the first application of a GD microchip in flow analysis. We have demonstrated the feasibility of using this GD-FIA system for measuring ammonia concentrations in environmental water samples thus providing proof of concept that complex sample pretreatment operations such as gas-diffusion separation can be conducted on a microchip platform. A limit of detection of $0.10 \mathrm{mg} \mathrm{L}^{-1} \mathrm{NH}_{3}$ and a relative standard deviation of $<5 \%$ were obtained. Since the sample bands were broad $(>100 \mathrm{~nL})$, the limit of detection may be further improved by employing a Z-shaped flow cell. Because the EOP rates were relatively low, the sample throughput was not high; this issue can be addressed by increasing the pump rates or minimizing the fluidic channels. Although two basic functional components were microfabricted in this work, a regular FIA injection valve and a conventional CE absorbance detector were employed for assembling the GD-FIA system. We are currently developing micro injection valves and miniaturized detectors and expect to build a microchip GD-FIA system before long. The major advantages of a microchip GD-FIA device will be the instrument portability, low production and consumable costs, and low waste generation. We expect that such a device will have many applications in the determination of volatile or semi-volatile analytes (e.g. low molecular mass phenols and alcohols) or analytes that can be converted into volatile chemical species (e.g. $\left.\mathrm{CO}_{3}{ }^{2-}, \mathrm{SO}_{3}{ }^{2-}, \mathrm{CN}^{-}\right)$.

Acknowledgments. This work is partially sponsored by the Department of Energy (DE-SC0006351), National Science Foundation (CHE 1011957), and the National Institutes of Health (R21GM104526). The authors are also grateful to the Australian Research Council for financial support (ARC Linkage Project 110200595). 


\section{References}

1. R̉užička J, Hansen E H (1975) Flow injection analyses: Part I. A new concept of fast continuous flow analysis. Anal. Chim. Acta, 78(1): 145-157.

2. Li H-F, Lin J-M (2009) Applications of microfluidic systems in environmental analysis. Anal. Bioanal. Chem., 393(2): 555-567.

3. Jokerst J C, Emory J M, Henry C S (2012) Advances in microfluidics for environmental analysis. Analyst, 137(1): 24-34

4. Ohira S-I, Toda K (2005) Micro gas analysis system for measurement of atmospheric hydrogen sulfide and sulfur dioxide. Lab Chip, 5(12): 1374-1379.

5. Cerdà A, Oms M T, Forteza R, Cerdà V (1995) Evaluation of flow injection methods for ammonium determination in wastewater samples. Anal. Chim. Acta, 311(2): 165-173.

6. Ružička J, Marshall G D (1990) Sequential injection: a new concept for chemical sensors, process analysis and laboratory assays. Anal. Chim. Acta, 237(0): 329-343.

7. Luque de Castro M D (2008) Membrane-based separation techniques: dialysis, gas diffusion and pervaporation, in Kolev S D, McKelvie I D Eds., Advances in Flow Injection Analysis and Related Techniques, Volume 54 Comprehensive Analytical Chemistry, Elsevier, Amsterdam, pp 203-232.

8. Kolev S D, Fernandes P R L V, Satinsky D, Solich P (2009) Highly sensitive gas-diffusion sequential injection analysis based on flow manipulation. Talanta, 79(4): 1021-1025.

9. Health effect information.

http://public.health.oregon.gov/HealthyEnvironments/DrinkingWater/Monitoring/Documents/health/ ammonia.pdf. Access 17 June 2014

10. Summary review of health effects associated with ammonia. http://www.epa.gov/iris/subst/0422.htm. Access 05 October 2014.

11. Oliveira S M, Lopes T I M S, Toth I V, Rangel A O S S (2009) Determination of ammonium in marine waters using a gas diffusion multicommuted flow injection system with in-line prevention of metal hydroxides precipitation, J. Environ. Monit. 11: 228-234.

12. Segundo R A, Mesquita R B R, Ferreira M T S O B, Teixeira C F C P, Bordalob A A, Rangel A O S S (2011) Development of a sequential injection gas diffusion system for the determination of ammonium in transitional and coastal waters. 3: 249-255.

13. Liu S, Pu Q, Lu J J (2003) Electric field-decoupled electroosmotic pump for microfluidic devices. J. Chromatogr. A, 1013(1-2): 57-64.

14. Pu Q, Liu S (2004) Microfabricated electroosmotic pump for capillary-based sequential injection analysis. Anal. Chim. Acta, 511(1): 105-112.

15. Byun C K, Wang X, Pu Q, Liu S (2007) Electroosmosis-Based Nanopipettor. Anal. Chem., 79(10): 3862-3866.

16. Wang W, Gu C, Lynch K B, Lu J J, Zhang Z, Pu Q, et al. (2014) High-Pressure Open-Channel On-Chip Electroosmotic Pump for Nanoflow High Performance Liquid Chromatography. Anal. Chem., 86(4): 1958-1964.

17. Liu S, Dasgupta P K (1994) Sequential injection analysis in capillary format with an electroosmotic pump. Talanta, 41(11): 1903-1910.

18. Satterfield M B, Majsztrik P W, Ota H, Benziger J B, Bocarsly A B (2006) Mechanical properties of Nafion and titania/Nafion composite membranes for polymer electrolyte membrane fuel cells. J. Polym. Sci., Part B: Polym. Phys., 44(16): 2327-2345.

19. Frank M J W, Kuipers J A M, van Swaaij W P M (1996) Diffusion Coefficients and Viscosities of $\mathrm{CO}_{2}+\mathrm{H}_{2} \mathrm{O}$, $\mathrm{CO}_{2}+\mathrm{CH}_{3} \mathrm{OH}, \mathrm{NH}_{3}+\mathrm{H}_{2} \mathrm{O}$, and $\mathrm{NH}_{3}+\mathrm{CH}_{3} \mathrm{OH}$ Liquid Mixtures. J. Chem. Eng. Data, 41(2): 297-302. 